\begin{tabular}{|l||c|c||}
\hline \hline Received 11.07.2021 & & JOTS \\
\hline \hline Accepted 19.07.2021 & Research Article & $5 / 2$ \\
& & $2021: 403-411$ \\
\hline \hline Published 24.07.2021 & & \\
\hline
\end{tabular}

\title{
Der Einleitungsśloka zur „Lehrschrift über das Eintreten in den Abhidharma" (T. 1554)
}

The introductory Śloka to "the Treatise on Entering the Abhidharma" (T. 1554)

\author{
Peter ZIEME* \\ Berlin/Germany \\ E-mail:ziemepet@gmail.com
}

The fragment Mainz 857 (verso) of the Berlin Turfan Collection contains a parallel to text $\mathrm{F}$ of the miscellaneous manuscript U 5335 (edited Berliner Turfantexte 34). The vertical lines (columns), which comprise an Old Uyghur translation of the beginning of the prologue to the Abhidharmāvatāra (T. 1554: 入阿毘達磨論 ru apidamo lun), are arranged in such a way that they proceed from right to left, contrary to the usual left-right direction.

Key Words: Prologue of the Abhidharmāvatāra, ancient Uyghur translation, script direction, Turfan Collection.

ORCID ID: 0000-0002-8090-7707. 


\section{Die Sammelhandschrift U 5335}

Die Sammelhandschrift U 5335 der Berliner Turfansammlung enthält kurze altuigurische Texte sowie chinesische Extrakte aus einer Vielzahl von Texten in uigurischer Umschrift der Hanzi-Zeichen, die bisweilen auch mit altuigurischer Übersetzung versehen wurden (BT 34). Letztere sind somit bilinguale Texte in einer Schrift. Den Fokus der Edition bildet die Rekonstruktion der Aussprache der chinesischen Zeichen, nachdem die meisten Texte der Sammelhandschrift identifiziert waren. Die Autoren haben zehn Texte dieser Sammelhandschrift identifiziert: B-a, D-a, E-a, E-b, F, G-a, J, N, O, und Q (BT 34: 50-51). Der Text A ist nicht bilingual, sondern nur altuigurisch (BT 34: 51-52). B-b ist ein Verehrungstext, der quasi auch identifiziert ist, wenn auch nicht eindeutig einer chinesischen Vorlage zugeordnet werden kann (BT 34:53-54). Der Text $\mathrm{C}$ ist ein rein altuigurischer Text (BT 34: 54-57). D-b ist ein kurzer Text eines Reuebekenntnisses und enthält die Namen der 10 Unterweltskönige (BT 34: 5859). ${ }^{1} \mathrm{G}-\mathrm{b}, \mathrm{G}-\mathrm{c}, \mathrm{H}$ und I-a, I-b, I-c, K, L, M P ${ }^{2}, \mathrm{R}^{3}$ sind weitestgehend bestimmt, jedoch nicht eindeutig lokalisierbar. Der Text $\mathrm{S}$ beschließt mit einem monolingualen altuigurischen Text diese Sammelhandschrift. Vorderhand ist nicht klar, weshalb gerade die ausgewählten Text(teil)e zusammengestellt wurden. Naheliegend ist es, an eine Art Übungsheft zu denken. Auf jeden Fall ist die Handschrift ein wichtiges Zeugnis dafür, welche Texte so im Umlauf waren. Dennoch fehlt uns für die getroffene Auswahl ein eindeutiger Grund, es mag einfach Zufall sein. Auf jeden Fall gibt uns die Handschrift einen Einblick in die Werkstatt eines altuigurischen Buddhisten.

\section{Text F der Sammelhandschrift}

Als Text F wurde der Abschnitt auf Seite 01 ZZ. 07 bis 16 ediert. Dieser Eingangsśloka zum Abhidharmāvatāra (T. 1554) eröffnet einen Prolog von

\footnotetext{
Die Autoren verweisen auf Dx 12367 der Dunhuang-Sammlung des IOM in St. Petersburg [Abb. 1]. Der Text umfaßt 10 Zeilen des 三十七尊禮懺文 sanshiqizun lichanwen und eine bilinguale Zeile: lenkadu sinkay $k(1)$ ya bitiyü tägintim ödig bolzun „Ich Lenkadu Sinkay-k(1)ya, habe es ergebenst geschrieben. Möge es aufgezeichnet bleiben!“. Der Name Lenkadu ist auch in der Mogao-Grotte 108 belegt, vgl. Matsui, 2017: 28, Lenkadu Inal. Den Namen erklärt Matsui als eine Wiedergabe von 蓮華奴, Sklave des Lotos:

Zum Text P vgl. Zieme 2020.

Zum Text P vgl. Zieme 2020.
} 
großer Ausstrahlungskraft, zumindest bei den uigurischen Buddhisten. Deren Zeugnisse lassen vermuten, daß dieser Abhidharmatext weite Verbreitung gefunden hatte. ${ }^{4}$

\section{Mainz 857 der Berliner Turfansammlung}

Die Rückseite des hier zu besprechenden achtzeiligen Fragments Mainz $857^{5}$ ist eine Variante dieses Ślokas, die zwei Besonderheiten aufweist.

1. Nach dem Vorbild von Sanskrittexten und in Übereinstimmung mit den chinesischen Verseinheiten verwendet der Schreiber am Ende einer Halbstrophe Dandas, doch ein Doppeldanda ist nicht vorhanden. Kasai hat zu Recht den grundsätzlichen Unterschied zwischen den zwei Abhidharma-Traditionen, einer sanskrit-tocharischen einerseits und einer chinesischen andererseits, bei den alten Uiguren (2021: 24) betont, doch hier zeigt sich eine Spur, daß die der chinesischen Tradition folgenden Abhidharma-Spezialisten durchaus auch mit Sanskrit-Handschriften vertraut gewesen sein könnten.

2. Besonders bemerkenswert ist, daß die Kolumnen von rechts nach links verlaufen, denn nur auf diese Weise läßt sich der erhaltene Text verstehen. Das erste Beispiel einer eben solchen Kolumnenrichtung ist das längere Fragment des Wutaishanzan in der Serindia-Sammlung von St. Petersburg (Zieme 2021). Die Kolumnen sind also von rechts nach links zu lesen. Diese Leserichtung steht im Gegensatz zur üblichen Weise, der zufolge man die Kolumnen von links nach rechts folgen lassen muß. Bei diesem Fragment könnte man meinen, daß der Schreiber der Kolumnenrichtung des chinesischen Textzitats folgen wollte. Da dies im Falle des Wutaishan-Gedichts nicht in Frage kommt, sollte man den zweiten Fall also auch zumindest ernst nehmen. Allerdings bleiben die beiden Beispiele eher Ausnahmen, sie bezeugen nicht, daß eine grundsätzliche Kolumnenrichtungsreform beabsichtigt war.

Shōgaito, 2014: 10-11; Shōgaito et al., 2018; Zieme, 2014.

5 Keine alte Signatur vorhanden. Die Vorderseite des Fragments trägt den Abschnitt T.XXV.1509.0462c03-18. 
Der Text von Mainz 857 verso [Abb. 2]

01 [敬禮一切智佛日無垢輪 ${ }^{6}$

02 [言光破]人天 惡趣本心闇

03 [agırın ay]agın yükünürm(ä)n tükäl bilgä

04 [biligligkä |] burhan-lıg kün t(ä)yri kirsiz

05 [tilgän]lig-kä | sav-lıg sözlüg

06 [yaruk] üzä buzdı tarkardı ärsär

07 [yalyuklarnıy tä]yri-lär-nin | üč tamu-nuy

08 [köyüldäki karaygusın |] t(ä)yri t(ä)yrisi sarvanyan

\section{Übersetzung}

Mit [tiefer Verehrung] verneige ich mich vor dem vollkommen weisen Buddha (=) dem schmutzlosen Sonnenrad. Durch [den Glanz] der Worte und Reden hat er zerstört und vernichtet bei [Menschen] und Göttern [die Finsternis] der drei Höllen. Der Göttergott, der Allweise (sarvajña) [...]

Vergleich von Mainz 857 verso mit dem Text F von U 5335

\begin{tabular}{|c|c|c|c|}
\hline & T.28.1554.0980b24-26 & Mainz 857 & U 5335 Text $\mathrm{F}$ \\
\hline 01 & 敬禮一切智 & & kyly ’yr sy cy \\
\hline 02 & 敬禮 & [agırın ay]agın yükünürm(ä)n & $\begin{array}{l}\text { agırın ayagın yükünü } \\
\text { täginürm(ä)n }\end{array}$ \\
\hline 03 & 一切智 & tükäl bilgä [biligligkä] & $\begin{array}{l}\text { s(a)rvartyan tükäl bilgä } \\
\text { biligligkä }\end{array}$ \\
\hline 04 & 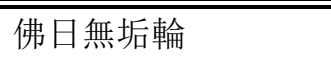 & & vyr šyr ’w äyv lwn \\
\hline 05 & 佛 & burhan-lig & burhan-lig \\
\hline 06 & 日無垢輪 & kün t(ä)yri kirsiz [tilgän]ligkä & $\begin{array}{l}\text { kün t(ä)yri kirsiz } \text { arıg } \\
\text { tilgänligkä }\end{array}$ \\
\hline 07 & 言光破人天 & & [k]yn qww p`šyn tyn \\
\hline 08 & 言 & savlıg sözlüg & sav-lug \\
\hline 09 & 光 & [yaruk] üzä & yaruk üzä \\
\hline 10 & 破 & buzd1 tarkardi ärsär & buzdači-ka \\
\hline
\end{tabular}

Erhalten sind nur wenige Reste der Hanzi-Zeichen. Einigermaßen rekonstruierbar ist das letzte Zeichen der Zeile 01 als lun 輪, doch ist nur noch der linke Bestandteil erkennbar. 


\begin{tabular}{|c|c|c|c|}
\hline 11 & 人天 & [yalyoklarnıy tä]yrilärniy & yalyoklı t(ä)yrili \\
\hline 12 & 惡趣本心闇 & & "q sww pyn sym 'ym \\
\hline 13 & 惡趣 & üč tamunuy & ayıg ažun-nuy \\
\hline 14 & 本心 & [töz köyüldäki (?)] & tözi bolmıš köyüldäki \\
\hline 15 & 闇 & [karaygu kararıgların] & karaygu kararıgların (Ende) \\
\hline 16 & ? 一切智言藏 & $\begin{array}{l}08 \text { t(ä)yri t(ä)yrisi sarvanyan } \\
\text { (der Text bricht hier ab) }\end{array}$ & \\
\hline
\end{tabular}

Das Stockholmer Fragment 1935.52.21 enthält einen detaillierten Kommentar über den Prolog, der bereits zweimal ediert wurde. (Elmalı 2015; Shōgaito et al., 2018) Darin werden ganz genau übereinstimmende Satzteile analysiert wie ayayu agırlayu yükünürm(ä)n tükäl bilgä biligkä und burhan-lıg kün t(ä) yri kirsiz arıg tilgänlig. Auch für die anderen Teile werden Synonyme und Erklärungen vorgebracht, ohne ganz genau den Wortlaut zu kopieren. Dieser Kommentar vermittelt, wie uigurische Kommentatoren den Text grammatisch und inhaltlich analysierten, wobei besonderer Wert auf die Metaphorik gelegt wurde, wozu die Termini yöläšürüg (HWAU: 915a) und nizay (HWAU: 492b) verwendet wurden.

\section{Prolog}

Die Zeilen, wie sie in der Tabelle dargestellt sind, bilden den Anfang des Prologs zum Abhidharmāvatāra (Abhidharmāvatāraśāstra' $)^{8}$ (T. 1554 入阿毘達 磨論 ru apidamo lun $)^{9}$, dessen Autor Skandhila ${ }^{10}$ war. Dieser Prolog besteht aus drei Strophen zum Triratna (Buddha, dharma, samgha). Zitiert seien hier die französische und die englische Übersetzung:

"Je rends hommage (namaskaromi) au Buddha omniscient (sarvajña), le soleil au disque immaculé (vimala) dont les rayons sont paroles qui détruisent

7 Rekonstruktion nach tibetisch Rab tu byed pa chos mnion pa la hyug pa shes bya ba = Abhidharmāvatāraprakaraṇa, vgl. Inokuchi, 1961: 336.

8 Zum chinesischen Titel vgl. Hōbōgirin: 132; Dhammajoti, 2008: 8. Es gibt keine komplette SanskritVersion, vgl. aber Matsuda 1996. Zu Fragmenten in Tocharisch A vgl. Inokuchi, 1961:336; Kudara 1980; Kudara 1982.

9 T.XXVIII.1554.0980b21-0989a19.

10 T.XXVIII.1554.0980b24. 


\section{1)}

chez hommes et dieux les racines des mauvaises destinées (durgatimūla) et l'obscurité de la pensée (cittāndhakāra) “11.

„Homage to the Omniscient One (sarvajña), / the Buddha-Sun, The Taintless Disc / Whose Words' radiance doth shatter / The mind's darkness of gods and men, / The Source of evil abodes." ${ }^{2}$

\section{Weitere Bemerkungen (nach den Zeilen der Tabelle)}

02 yükünürm(ä)n. Der so rekonstruierten Lesung liegen die Buchstaben ywkwnrmn zugrunde. Es handelt sich im ganzen um eine recht flüchtige Handschrift, es war wahrscheinlich nur eine Art Konzept.

06 Zur Metapher Buddha-Sonne (skt. tathāgatāditya) (Kaygusuz, 2021: 118122) vgl. 問仏-lig 日天-kä tep tedi „Frage: Warum sagt man Buddha-Sonnengott?“; 答日天 ärsär yöläšürüg ol „Antwort: Was Sonnengott betrifft, so ist es eine Metapher" (Shōgaito et al., 2018: 4 [21R2]).

10 Warum hier ärsär steht, ist nicht klar. In U 5335 steht ein Dativsuffix.

13 üč tamu ,drei Höllen'statt, (drei) schlechte Wege' (durgati) ist eine ad hoc-Bildung, die von einer Vorlage nicht erklärbar ist. Die drei schlechten Existenzen sind die der Höllen, der Pretas und der Tiere. Eine korrekte Übersetzung von equ 惡趣 hat U 5335 mit ayıg ažun, schlechte Existenz؛

16 Wie diese Zeile zu lokalisieren ist, bleibt unklar. Das letzte Wort der Zeile ist eine fehlerhafte (?) kurze Schreibform für sarvayatan « skt. sarvajña ,allwissend' (HWAU: 588b), was meist durch tükäl bilgä biliglig oder alkunı bildäči übersetzt wird. Die Wörter dieser Zeile 08, mit denen die Aufzeichnung abrupt abbricht, könnten den Anfang des ,Dharma'-Śloka gebildet haben: 一切智言藏 ,Wort-Schatz des All-Wissenden“ = , [Homage to] the Basis / Of the Omniscient's Words' (Dhammajoti, 2008: 1); ,[Moi je salue de la tête un tel] trésor de paroles omniscientes.' (Velthem, 2008: 1)

\section{Abkürzungen}

BT 34 = Shōgaito et al., 2015

\footnotetext{
11 T.XXVIII.1554.0980b24-26. Übersetzung Velthem 1977: 1.

12 Dhammajoti, 2008: 71. [ausführliche Rezension von Kritzer 2010]
} 


\section{0}

DDB $=$ Digital Dictionary of Buddhism (Ed. Charles Muller).

HWAU = Wilkens, 2021.

T. = Taishō Shinshū Daizōkyō.

\section{Literaturverzeichnis}

Dhammajoti, K. L. (2008). Entrance into the Supreme Doctrine: Skandhila's Abhidharmāvatāra. University of Hong Kong.

Elmalı, M. (2015). Eski Uygurca Gramer Terimleri: vibakti-samaz. Kesit Yayınları.

Hōbōgirin (1978). Dictionnaire encyclopédique du bouddhisme d'après les sources chinoises et japonaises. Publié par l'Académie des Inscriptions et Belles-Lettres. Institut de France avec le patronage de l'Académie du Japon et le concours de l'École Française d'Extrème Orient et de la Maison Franco-japonaise de Tokyo, I-VII (1929-1994). Répertoire du Canon bouddhique sino-japonais.

Inokuchi, T. (1961). トカラ語及びウテン語の佛典 IV [Tocharische und khotanische Buddhica IV]. 西域文化研究 [Monumenta Serindica]. Hōzōkan.

Kasai, Y. (2021). Old Uyghur Translations of Buddhist Texts and their Usage. In Brentjes, von S. \& Fidora, A. (Eds.), Contact and transmission. Intercultural encounters from late antiquity to the early modern Period II (pp. 13-31). Brepols Publishers.

Kaygusuz, A. (2021). Buddhistische Bildersprache in alttürkischen Texten. Eine literaturwissenschaftliche und philologische Analyse. Harrassowitz Verlag.

Kritzer, R. (2010). Entrance into the Supreme Doctrine: Skandhila's Abhidharmāvatāra, 2nd rev. ed. by K.L. Dhammajoti. Indo-Iranian Journal, 53(3), 265-274. (Rezension).

Kudara, K. (1980). 入阿毘達磨論の註釋書について [Zum Kommentar des Abhidharmāvatāra-prakaraṇa]. 印度學佛教學研究 [Journal of Indian and Buddhist Studies], 29(1), 72-77.

Kudara, K. (1982). ウイグル訳アビダルマ論書に見える論師・論書の梵名 [Sanskritische Namen von Śāstra-Meistern und Śāstra-Werken nach dem uigurischen Abhidharmaśāstra]. 印度学仏教学研究 [Journal of Indian and Buddhist Studies], 31, 112115.

Matsuda, K. (1996). 『入阿毘達磨論』のギルギット写本残關 [Sanskrit Fragment of the Abhidharmavatara in the Gilgit Manuscripts]. 佛教大学仏教学会紀要 [Bulletin of the Association of Buddhist Studies Bukkyo University], 4, 35-42. 


\section{0}

Matsui, D. (2017). 松井太 敦煌石窟ウイグル語 モンゴル語題記銘文集成 [Uigur and Mongol Inscriptions of the Dunhuang Grottoes]. In Matsui, D. \& Arakawa, S. (Eds.), 敦煌石窟多言語資料集成 [Multilingual Source Materials of the Dunhuang Grottoes] (pp. 1-162). Research Institute for Languages and Culture of Asia and Africa.

Shōgaito, M. (2004). ウイグル文「入阿毘達磨論」の注釈書断片 [Ein uigurisches Fragment des Kommentars zum Abhidharmāvatāra-prakaraṇa]. Contributions to the Studies of Eurasian Languages, 7, 271-295.

Shōgaito, M. (2013). An Uighur fragment of the 'Commentaries on abhidharmakośakārikā (俱舎論頌疏)'. Abant İzzet Baysal Üniversitesi Sosyal Bilimler Enstitüsü Dergisi, 13, 341-351.

Shōgaito, M. (2014). The Uighur Abhidharmakośabhāṣya preserved at the Museum of Ethnography in Stockholm. Harrassowitz Verlag.

Shōgaito, M. et al. (2015). The Berlin Chinese Text U 5335 Written in Uighur Script. Berliner Turfantexte 34. Brepols Publishers.

Shōgaito, M. et al. (2018). The Uigur version of the commentary on Abhidharmaprakarana-śāstra preserved at the Museum of Ethnography in Stockholm. In Hayasi, T. et al. (Eds.), ユーラシア諸言語の多様性と動態 - 20 号記念号一追悼 庄垣内正弘先生 [Diversity and Dynamics of Eurasian Languages. The 20th Commemorative Volume. Dedicated to Prof. Dr. Masahiro Shōgaito], 20, 1-68.

Velthem, van M. (1977). Le traité de la descente dans la profonde loi (Abhidharmāvatāraśāstra) de l'Arhat Skandhila. Louvain-la-Neuve.

Wilkens, J. (2021). Handwörterbuch des Altuigurischen, Altuigurisch-Deutsch-Türkisch. Universitätsverlag Göttingen.

Zieme, P. (2014). Ein chinesisch-altuigurischer Abschnitt aus dem „Traktat über das Eintreten in den Abhidharma" (Abhidharmāvatāra). Türk Dilleri Araştırmaları, 24(2), 305-314.

Zieme, P. (2020). Buddhist pāramitās as seen from Old Uygur texts. Annual Report of the International Research Institute for Advanced Buddhology (ARIRIAB) at Soka University for the Academic Year 2019, 22, 147-165.

Zieme, P. (2021). Mount Wutai and Mañjuśrī in Old Uigur Buddhism. In Andrews, S. et al. (Eds.), The Transnational Cult of Mount Wutai. Historical and Comparative Perspectives (pp. 223-237). Brill. 


\section{)(ङ)}

\section{Tafeln}

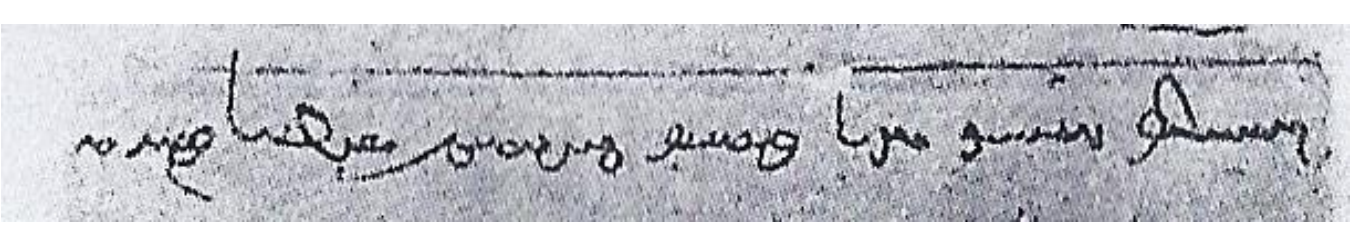

Abb. I

Дх 12367



Abb. II

Mainz 857 verso 